\section{AB0322 DEVELOP AN MASTER ALGORITHM FOR DRUG WITHDRAW STRATEGY IN REDUCTION OF ADVERSE EVENTS WITH COMBINATION THERAPY- A MACHINE LEARNING MODEL FROM THE SMART SYSTEM OF DISEASE MANAGEMENT (SSDM)}

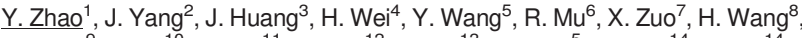

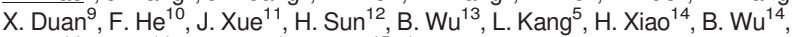
Y. Liu ${ }^{14}$, Y. Jia ${ }^{14}$, F. Zhang ${ }^{1}$, F. Xiao ${ }^{15} .{ }^{1}$ Department of Rheumatology and Immunology, Peking Union Medical College Hospital, Beijing; ${ }^{2}$ Department of Rheumatology, Central Hospital of MianYang, Sichuan, Mianyang; ${ }^{3}$ Department of Rheumatology and Immunology, The Sixth Affiliated Hospital of Sun Yat-sen University, Guangzhou; ${ }^{4}$ Department of Rheumatology, Northern Jiangsu People's Hospital, Yangzhou; ${ }^{5}$ Department of Rheumatology and Immunology, The First Affiliated Hospital of BaoTou Medical College, Baotou; ${ }^{6}$ Department of Rheumatology and Immunology, People's Hospital, Beijing University Medical School, Beijing; ${ }^{7}$ Department of Rheumatology and Immunology, Xiangya Hospital Centeral South University, Changsha; ${ }^{8}$ Department of Rheumatology and Immunology, JiaXing First Hospital, Zhejiang, Jiaxing; ${ }^{9}$ Department of rheumatology, The Second Affiliated Hospital of Nanchang University, Nanchang; ${ }^{10}$ Department of Rheumatology, Suining Central Hospital, Suining; ${ }^{11}$ Department of Rheumatology and Immunology, The Second Affiliated Hospital of Zhejiang University School of Medicine, Hangzhou; ${ }^{12}$ Department of rheumatology, Shandong Provincial Hospital Affiliated to Shandong University, Jinan;

${ }^{13}$ Department of Rheumatology, Chongqing Hospital of Traditional Chinese Medicine, Chongqing; ${ }^{14}$ Shanghai Gothic Internet Technology Co., Ltd., Shanghai; ${ }^{15}$ Suzhou Antiinflammatory Research Institue, Suzhou, China

Background: Combination therapy with DMARDs for treating RA is considered as standard of care. However, certain rates of adverse events (AEs) are unavoidable. The stigma is which drug should be stopped first once AEs emerge. The decisions made by clinicians are always empirically.

Objectives: To develop an algorithm for decision making on drug withdraw sequence in face of adverse events with combination therapy based on data mining and machine learning from the SSDM.

Methods: SSDM is an interactive mobile disease management tool, including the doctors' and patients' application system (App). The patients can input medical records and perform self-evaluation via App. The data synchronises to the mobiles of authorised rheumatologists through cloud and advices could be delivered. In order to develop the master algorithm, abnormal white blood cell (WBC) counts in blood were first targeted. WBC and medication data was collected, extracted, validated, and then based on Bayesian networks, data mining, modelling, calculating, analysing were performed. WBC under $4,000 / \mathrm{ml}$ is defined as leukocytopenia (LP), and over $10,000 / \mathrm{ml}$ as infection predisposing (IP).

Results: From Jun 2014 to Jan 2018, 24,731 RA patients from 486 centres registered in SSDM. 6099 are male and 18632 are female with mean age of 49.28 \pm 16.08 (18 to 99) years. 19 different drugs and 126 types of combination therapies are identified. Lab test results showed LP happened in 87 and IP in 123 treatment regiments. Among them we selected prednisone (Pred), leflunomide (LEF), methotrexate (MTX) and hydroxycholoqine (HCQ) as an example to develop a master algorithm based on Bayesian networks and learning model. Figure 1 shows Bayesian network and data processing, in which, quartet are correlating with 15 different regiments. Based on Bayesian method and network data, the calculation for LP and IP probabilities is generated through 32 modelling, and the algorithm for drug withdraw strategies are generated. Drug withdraw sequence for LP is HCQ, then LEF and then Pre, and the risks of LP are reduced by $64 \%, 52 \%$ and $26 \%$, respectively. For IP, withdraw sequence is Pred, then MTX and then $\mathrm{LEF}$, and the risks of IP are reduced by $57 \%, 63 \%$, and $14 \%$, respectively.

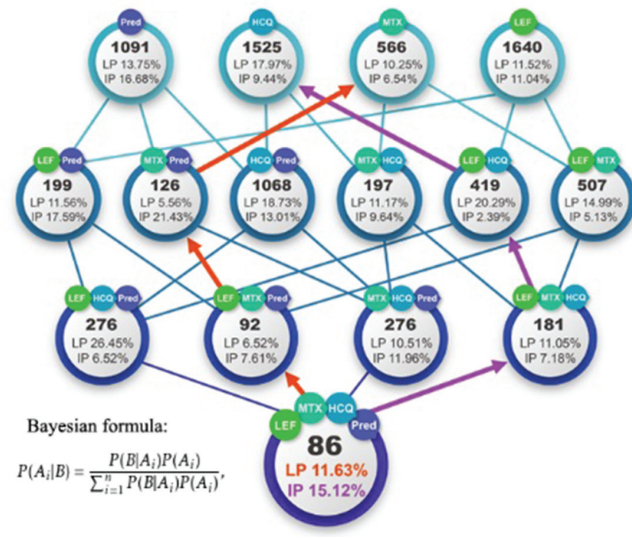

Abstract AB0322 - Figure 1. Bayesian network and data processing: patients' number (black bold as blow showed) and the rate of either LP or IP in 15 regiments.
Pred: prednisone; HCQ: hydroxycholoqine; MTX: methotrexate; LEF: leflunomide; LP: leukocytopenia; IP: infection predisposing

Conclusions: Big data system can be built using SSDM via empowering patient. Through data mining, networking, modelling, and Bayesian calculation, a master algorithm for drug withdraw strategy in reduction of adverse events with combination therapy is developed, which can be applied on the other AEs in SSDM and may replicated in other diseases. Following the continuing data inputs and machine leaning, an artificial intelligent system in assisting clinical decision making may be achieved.

Limitations: This study only focus on rate of AE without considering the efficacy, without stratifying dosing.

Disclosure of Interest: None declared

DOI: 10.1136/annrheumdis-2018-eular.4063

\section{AB0323 RELATIONSHIB BETWEEN SERUM LEVELS OF LEPTIN \& HOMOCYSTEIN IN RHEUMATOID ARTHRITIS PATIENTS WITH OR WITHOUT EXTRA ARTICULAR MANIFESTATIONS}

\section{Y. Hussein Gazar. Rheumatology Department, Al-Azhar University, Cairo, Egypt}

Background: Rheumatoid arthritis (RA) is a chronic, systemic disorder with unknown etiology, which is characterised by involvement of hand joints and deformation. Rheumatoid arthritis is characterised by synovial inflammation and hyperplasia. chronic inflammatory process of the joints that progresses through different stages of increasing severity characterises rheumatoid arthritis.

Objectives: In this study we aimed to determine the relationship and correlation between serum homocystein and leptin levels in (patients with rheumatoid arthritis (RA) without extra articular manifestation (non Ex RA)(GROUP-A) and RA patients with extra articular manifestation (ExRA) (GROUP B) and disease progression.

Methods: 80 patients diagnosed as rheumatoid arthritis (according TO ACR/ EULAR 2010 classification criteria) (according TO ACR/EULAR 2010 classification criteria) and selected as follow:

(Group A)

40 patients without extra articular manifestation (nonExRA) and

(Group B)

40 patients with variable extra articular manifestation (ExRA)),

CONTROL GROUP $(\mathrm{C})$

Another 40 apparently healthy persons as controls.

RA patients were divided into three groups based on Disease Activity Scores in 28 joints (DAS28) as low disease activity, moderate disease activity, and high dis ease activity groups.

Of the patients (non Ex RA), 11 (27.5\%) had low disease activity (DAS28=2.6$3.2), 15(37.5 \%)$ had moderate disease activity (DAS28=3.2-5.1), and $14(35.0 \%)$ had high disease activity (DAS28 $\geq 5.1$ ). Laboratory investigations were performed for all patients, including determination of haemoglobin concentration $(\mathrm{Hb})$, erythrocyte sedimentation rate (ESR), and C-reactive protein. Serum concentrations of tumour necrosis factor-alpha (TNF- $\alpha$ ), IL-6, Homocystein and Leptin were measured.

Results: Significant differences were found between RA patients (group A+group $\mathrm{B}$ ) and controls healthy group (group $\mathrm{C}$ ) with regard to the mean levels of $\mathrm{Hb}$, ESR, TNF- $\alpha$ and IL-6 $(\mathrm{p}<0.05)$.

As regards to serum leptin, non significant level differences between healthy control group $(20.43 \pm 8.73 \mathrm{ng} / \mathrm{ml}$ ) and patient groups (group II and III) (22.43 $\pm 7.73 \mathrm{ng} / \mathrm{ml})$.

while A statistically significant higher mean level serum Homocystein concentration $(p<0.05)$ was found in patients (group $A$ and $B)(11.79+8.72 \mu \mathrm{mol} / L)$ than in control (group C) $(8.8+1.58 \mu \mathrm{mol} / \mathrm{L})$.

Significant differences were found between non ExRA (group A) and ExRA (group $B$ ) with regard to the mean levels of $\mathrm{Hb}$, ESR, TNF- $\alpha$ and IL-6 $(p<0.05)$.

A statistically non significant differences in mean level of serum Leptin concentration $(p>0.05)$ was found in group $A(22.43 \pm 5.73 \mathrm{ng} / \mathrm{ml})$ than in group B $(24.43$ $\pm 5.73 \mathrm{ng} / \mathrm{ml})$.

While a significant mean level of serum Homocystein concentration ( $P$ value $<0.05)$ was found in Group B patients $(19.43 \pm 1.08 \mu \mathrm{mol} / \mathrm{L})$ than in group $A$ $(11.79 \pm 13.05 \mu \mathrm{mol} / \mathrm{L})(\mathrm{p}<0.05)$.

Positive significant correlations were detected between serum Homocystein and ESR, TNF- $\alpha$, IL-6, and DAS-28 $(p<0.05)$ in group B

Multiple linear regression analysis showed that DAS- 28 and ESR were the main variables associated with serum Homocystein in RA patients $(p<0.05)$.

Conclusions: Serum leptin can't be considered of value as an inflammation marker in monitoring RA patients.

Seum homocystein can be used as a marker for probability of extra articular complication of RA.

Disclosure of Interest: None declared

DOI: 10.1136/annrheumdis-2018-eular.1226 